The Astrophysical Journal Supplement Series, 90:899-903, 1994 February

(c) 1994. The American Astronomical Society. All rights reserved. Printed in U.S.A.

\title{
GAMMA-RAY BURST SPECTRAL BREAKS AND SOURCE BEAMING
}

\author{
MATTHEW G. BARING ${ }^{1}$ \\ Laboratory for High Energy Astrophysics, Code 665, NASA Goddard Space Flight Center, Greenbelt, MD 20771 \\ Received 1993 March 8; accepted 1993 June 21
}

\begin{abstract}
The principal discovery of the Compton Gamma-Ray Observatory (GRO) concerning gamma-ray bursts is that these sources are isotropic but with a comparative deficiency of fainter sources, suggesting that they are probably cosmological in origin. If they are at such large distances from Earth then they are extremely luminous and compact. A consequence of this is that two-photon pair production attenuation of the gamma-ray continuum cannot be avoided unless the source radiation is substantially beamed. Most sources do not display gamma-ray turnovers although a few GRB detected by $G R O$ exhibit distinct spectral breaks in the $\mathrm{MeV}$ range. A derivation of the relationship defining of the degree of beaming in burst sources with spectral breaks due to $\gamma-\gamma$ attenuation, as a function of source spectral index and break energy, is presented. It is found that sources at distances of $\sim 1 \mathrm{Gpc}$ must typically be beamed with bulk Lorentz factors of around $10^{3}-10^{4}$, indicating powerful bulk acceleration in bursts, although these Lorentz factors are reduced markedly for steep source spectra. Since the source spectra are not strongly Comptonized, such beaming will blueshift the $\gamma-\gamma$ attenuation breaks to energies much higher than 1 $\mathrm{MeV}$; an absolute lower bound to the source bulk Lorentz factor is determined from this additional constraint. This blueshifting suggests that those sources with MeV breaks may not be cosmological, or that their breaks are produced by a mechanism that dominates $\gamma-\gamma$ attenuation at these energies.
\end{abstract}

Subject headings: gamma-rays: bursts — radiation mechanisms: nonthermal

\section{INTRODUCTION}

The observations of the BATSE experiment aboard GRO that the gamma-ray burst (GRB) population is isotropic but inhomogeneous with a comparative deficiency of weaker sources (Meegan et al. 1992) have dramatically bolstered the premise that most bursts are cosmological in origin (Mao \& Paczyński 1992a), rather than Galactic neutron stars (Mao \& Paczyński 1992b). There is no evidence that recent accumulations of burst data have changed this picture. In addition to this, BATSE has also observed definite spectral breaks (Schaefer et al. 1992) between $400 \mathrm{keV}$ and $2 \mathrm{MeV}$ in four (maybe five) bursts, while EGRET spectra for three of these bursts (see Schneid et al. 1992 for GRB 910503; Kwok et al. 1993 for GRBs 910601 and 910814 ) provide convincing supporting evidence for the breaks detected by BATSE.

Recently, the BATSE (see Kouveliotou et al. 1993) and EGRET (see Sommer et al. 1994) detections of the "Superbowl" burst GRB 930131 have revealed a possible small spectral break in this source at similar energies. Whether GRBs with breaks are a class of objects distinct from the majority that show no evidence of breaks (as yet) remains to be seen. An alluring fact is that perhaps all sources seen by EGRET have spectral breaks, so such sources may not necessarily be a minority. This provides ample motivation for examining the implications of breaks for GRB models.

The most popular mechanism invoked to explain gammaray spectral breaks is pair production $\gamma \gamma \rightarrow e^{+} e^{-}$, which is explored in detail in this paper. Two-photon pair production has long been recognized as a mechanism for attenuating gamma-ray spectra at energies above about $1 \mathrm{MeV}$ in a variety

\footnotetext{
${ }^{1}$ NAS-NRC Research Associate.
}

of astrophysical contexts (e.g., see Baring 1993 and references therein). This mechanism was first applied to gamma-ray bursts by Schmidt (1978), who proposed that it could be used to provide upper limits to the distance to sources that revealed no spectral breaks above $1 \mathrm{MeV}$. Considering only isotropic source photons, Schmidt concluded that since the bursts seen before then had no breaks, they must have been Galactic, in direct conflict with the recent BATSE results' implications for burst populations.

A well-known feature of $\gamma \gamma \rightarrow e^{+} e^{-}$is that its threshold energy $\chi$ in the center-of-momentum frame is strongly dependent on the angle $\Theta$ between the photon directions $\left[\chi^{2} \propto(1-\right.$ $\cos \Theta$ )]. This can dramatically reduce the optical depth of the process and suppress gamma-ray spectral breaks, thereby permitting a given GRB to be more distant from Earth (Schmidt 1978). Krolik \& Pier (1991) considered the effect of extreme radiation anisotropy on the pair production process for gamma-ray bursts; in extragalactic sources a high degree of beaming is necessitated, corresponding to relativistic bulk motions of the emitting plasma (Fenimore et al. 1992).

In this paper, the regime of relativistic beaming is explored, obtaining a relationship between photon-beaming angle, source distance and spectral break energy for $\gamma-\gamma$ attenuation of GRB continua. The implications of this constraint for $G R O$ sources are discussed by Baring (1993). It is observed that while this relationship confirms that extragalactic sources, and in particular cosmological ones, have extremely high radiation collimation, the deduced relativistic beaming is reduced markedly for steep source spectra. Since sources show no evidence of Comptonization, such beaming would blueshift the breaks to higher energies, causing problems for a $\gamma-\gamma$ attenuation interpretation of $\mathrm{MeV}$ spectral breaks in sources at cosmological distances. The resulting absolute lower limit on the bulk Lo- 
rentz factor in sources is obtained in equation (16). While the deduced relativistic beaming is compelling evidence for bulk acceleration in most GRBs, those sources with observed breaks probably have their breaks generated by a mechanism that dominates $\gamma-\gamma$ attenuation at $\mathrm{MeV}$ energies, whether these sources have relativistic bulk motion or not.

\section{PAIR PRODUCTION OPTICAL DEPTH}

The application of pair production in anisotropic environments to GRO gamma-ray bursts is made in Baring (1993); the relationships used there are derived in detail here. To assess the role of two-photon pair production in attenuating gammaray burst spectra, the interactions of photons created only within the emission region are considered here, neglecting the presence of any external radiation. If external radiation plays an important role then the details of pair production attenuation become very model dependent. For simplicity, the photon distribution is assumed to be cylindrically symmetric about some beaming axis (that is aligned near to the line of sight of a distant observer), with the spectrum $n(\epsilon)$ being identical for all photon angles $\theta_{\epsilon}$ relative to the axis. Here $\epsilon$ is the photon energy in units of $m_{e} c^{2}$, a convention adopted throughout this paper. The photon distribution function then takes the form $n_{\gamma}\left(\epsilon, \mu_{\epsilon}\right)=n(\epsilon) f\left(\mu_{\epsilon}\right)$, with $f\left(\mu_{\epsilon}\right)$ normalized to unity, and where $\mu_{\epsilon}=\cos \theta_{\epsilon}$. Further, for anisotropic (beamed) photons we take the simple case that the angular distribution about the axis is uniform within a cone of half-angle $\theta_{\varepsilon}=\theta_{m}$ (which can be dependent on the energy $\epsilon$-see below):

$$
f\left(\mu_{\epsilon}\right)=\frac{1}{1-\mu_{m}}, \quad \mu_{m} \equiv \cos \theta_{m} \leq \mu_{\epsilon} \leq 1 .
$$

This form suffices to describe the general nature of photon beaming effects on pair production; for substantial beaming it is a good approximation to an isotropic radiation field moving at some bulk velocity with respect to an observer.

For photons that interact with themselves to produce pairs, a form for the pair production optical depth $\tau_{\gamma \gamma}(\epsilon)$ can be formed using equation (7) of Stepney \& Guilbert (1983), who have performed the trivial angle integrations for photon distributions independent of azimuthal angles:

$$
\begin{array}{r}
\tau_{\gamma \gamma}(\epsilon)=\frac{4 R}{\pi} \int_{-1}^{1} d \mu_{\epsilon} f\left(\mu_{\epsilon}\right) \int_{-1}^{1} d \mu_{\omega} f\left(\mu_{\omega}\right) \int_{0}^{\infty} d \omega \frac{n(\omega)}{\epsilon \omega} \\
\times \int_{\chi_{-}}^{x_{+}} \frac{\chi^{3} \sigma_{\gamma \gamma}(\chi) d \chi}{\sqrt{\left(\chi_{+}^{2}-\chi^{2}\right)\left(\chi^{2}-\chi_{-}^{2}\right)}} .
\end{array}
$$

Here $\chi=[\epsilon \omega(1-\cos \theta) / 2]^{1 / 2}$ is the center-of-momentum frame (CM) energy of the photons, whose energies and angle cosines in the observer's frame are $\left(\epsilon, \mu_{\epsilon}\right)$ and $\left(\omega, \mu_{\omega}\right)$, respectively. $\Theta$ is the angle between the photon directions. The pair production threshold condition is then $\chi \geq 1$.

Note that equation (2) has included an extra factor $1 /\left(\pi^{2} \epsilon^{2} \omega^{2}\right)$ in the result of Stepney \& Guilbert (1983) due to the difference in definitions of $n_{\gamma}\left(\epsilon, \mu_{\epsilon}\right)$; two factors of $1 / 2 \pi$ must be included to normalize the photon phase angle distributions correctly, and two extra factors of 2 are needed because of the unit normalization of $f\left(\mu_{\epsilon}\right)$. Since $\mu_{\epsilon}=\cos \theta_{\epsilon}$ and $\mu_{\omega}=$ $\cos \theta_{\omega}$

$$
\chi_{ \pm}^{2}=\frac{\epsilon \omega}{2}\left[1-\cos \left(\theta_{\epsilon} \pm \theta_{\omega}\right)\right]
$$

For $\gamma-\gamma$ turnovers in burst spectra, it is appropriate to consider attenuation of an infinite power-law photon distribution, the simplest first approximation to a GRB spectrum:

$$
n(\epsilon)=n_{\gamma} \epsilon^{-(1+\alpha)}
$$

Inserting this and equation (1) into equation (2) and changing the order of integration gives an optical depth

$$
\tau_{\gamma \gamma}(\epsilon)=n_{\gamma} \sigma_{T} R \mathscr{H}(\alpha) \mathscr{F}_{\alpha}\left(\theta_{m}\right) \epsilon^{\alpha},
$$

with the angular distributions contributing the factor

$$
\begin{aligned}
\mathscr{\mathscr { F }}_{\alpha}\left(\theta_{m}\right)=\frac{1}{\pi} \int_{\mu_{m}}^{1} \frac{d \mu_{\epsilon}}{1-\mu_{m}} \int_{\mu_{m}}^{1} \frac{d \mu_{\omega}}{1-\mu_{m}} \\
\quad \times \int_{\zeta_{-}}^{\zeta_{+}} \frac{\zeta^{1+\alpha} d \zeta}{\sqrt{\left(\zeta_{+}-\zeta\right)\left(\zeta-\zeta_{-}\right)}} .
\end{aligned}
$$

where $\zeta_{ \pm}=\chi_{ \pm}^{2} / \epsilon \omega=\left[1-\cos \left(\theta_{\epsilon} \pm \theta_{\omega}\right)\right] / 2$. The $\zeta$ integral can be expressed in terms of Legendre functions $\mathscr{P}_{\nu}(\kappa)$, using the change of variables $2 \zeta=\left\{\zeta_{+}+\zeta_{-}+\left(\zeta_{+}-\zeta_{-}\right) \cos \phi\right\}$ and the identity 8.822.1 in Gradshteyn \& Rhyzik (1980), yielding

$$
\mathscr{F}_{\alpha}\left(\theta_{m}\right)=\int_{\mu_{m}}^{1} d \mu_{\epsilon} \int_{\mu_{m}}^{1} d \mu_{\omega}\left\{\frac{\left|\mu_{\epsilon}-\mu_{\omega}\right|}{2}\right\}^{1+\alpha} \frac{\mathscr{P}_{1+\alpha}(\kappa)}{\left(1-\mu_{m}\right)^{2}}
$$

for $\kappa=\left(1-\mu_{\epsilon} \mu_{\omega}\right) /\left|\mu_{\epsilon}-\mu_{\omega}\right|$. In equation (5),

$$
\mathscr{H}(\alpha)=\frac{4}{\sigma_{T}} \int_{1}^{\infty} d \chi \chi^{-(1+2 \alpha)} \sigma_{\gamma \gamma}(\chi) \approx \frac{7}{6(1+\alpha)^{5 / 3}},
$$

is an integration over the pair production cross section $\sigma_{\gamma \gamma}$.

The approximation in equation (8) was obtained using the result for $\mathscr{H}(\alpha) /(2+\alpha)$ given in equation (B6) of Svensson (1987, who also gives an exact result) and is accurate to better than $1 \%$ for $0<\alpha<6$. When the spectrum is flat with $\alpha=-1$, the integrations over $\mu_{\epsilon}$ and $\mu_{\omega}$ in equation (7) are trivial, yielding $\widetilde{\mathscr{F}}_{-1}\left(\theta_{m}\right)=1$. Polynomial expressions for $\mathscr{\mathscr { F }}_{\alpha}\left(\theta_{m}\right)$ are obtainable for integer $\alpha$; the first few are

$$
\begin{aligned}
& \mathscr{F}_{0}\left(\theta_{m}\right)=\frac{1}{8}\left(1-\mu_{m}\right)\left(3+\mu_{m}\right) \\
& \mathscr{F}_{1}\left(\theta_{m}\right)=\frac{1}{24}\left(1-\mu_{m}\right)^{2}\left(5+4 \mu_{m}+\mu_{m}^{2}\right), \\
& \mathscr{F}_{2}\left(\theta_{m}\right)=\frac{1}{256}\left(1-\mu_{m}\right)^{3}\left(35+47 \mu_{m}+25 \mu_{m}^{2}+5 \mu_{m}^{3}\right) .
\end{aligned}
$$

In the special case that the photons are isotropic, where $\theta_{m} \rightarrow \pi$, the form in equation (6) can be manipulated by changing variables to $\phi$ with $2 \zeta=\left[\zeta_{+}+\zeta_{-}+\left(\zeta_{+}-\zeta_{-}\right) \cos \phi\right]$. Further, if the cosine $\mu_{t \omega}=\cos \theta$ of the angle between the 
photons is used as a variable instead of $\mu_{\omega}$, then $\zeta=\left(1-\mu_{\epsilon \omega}\right) / 2$ and a solid angle transformation $\left(\mu_{\omega}, \phi\right) \rightarrow\left(\mu_{\epsilon \omega}, \phi_{\epsilon \omega}\right)$ results in a dramatic simplification of the three integrals:

$$
\mathscr{F}_{\alpha}(\pi)=\frac{1}{4 \pi} \int_{-1}^{1} d \mu_{\epsilon} \int_{-1}^{1} d \mu_{\epsilon \omega} \int_{0}^{\pi} d \phi_{\epsilon \omega} \zeta^{1+\alpha}=\frac{1}{2+\alpha} .
$$

This then produces an optical depth (eq. [5]) of

$$
\tau_{\gamma \gamma}(\epsilon)=n_{\gamma} \sigma_{T} R \frac{\mathscr{H}(\alpha)}{2+\alpha} \epsilon^{\alpha},
$$

which is equivalent to the isotropic result obtained by Gould \& Schreder (1967; see also eq. [B3] of Svensson 1987).

Of specific interest is the case where $\theta_{m} \ll 1$ and the radiation beaming in the observer's frame is large. This situation is expected to be appropriate for cosmological bursts because it reduces the optical depth of $\gamma \gamma \rightarrow e^{+} e^{-}$. It is then easily shown using substitutions of the form $1-\mu_{t, \omega}=\left(1-\mu_{m}\right)(s \pm t) / 2$ and a change of integration order, that $\mathscr{H}_{\alpha}\left(\theta_{m}\right)$ has a power-law dependence on $\theta_{m}$ :

$$
\mathscr{\mathscr { F }}_{\alpha}\left(\theta_{m}\right) \approx \frac{1}{3+\alpha}\left(\frac{\theta_{m}}{2}\right)^{2(1+\alpha)} \int_{1}^{\infty} d u\left(\frac{2}{1+u}\right)^{3+\alpha} \mathscr{P}_{1+\alpha}(u) .
$$

The identities 8.771.1 and 7.512.10 of Gradshteyn \& Ryzhik (1980) can be used to evaluate the integral, yielding

$$
\widetilde{F}_{\alpha}\left(\theta_{m}\right) \approx \mathscr{A}(\alpha) \theta_{m}^{2(1+\alpha)},
$$

where

$$
\mathscr{A}(\alpha)=\frac{2^{-(1+2 \alpha)} \Gamma(2 \alpha+4)}{\Gamma(\alpha+3) \Gamma(\alpha+4)} \approx \frac{2}{(7 / 3+\alpha)^{27 / 11}} .
$$

For $0<\alpha<6$, the approximation in equation $(12 b)$ is accurate to better than $1 \%$. The form in equation (12a) produces the dependence $\tau_{\gamma \gamma}(\epsilon) \propto \epsilon^{\alpha} \theta_{m}^{2(1+\alpha)}$, which arises because of pairproduction kinematics: the cross section peaks above the $\mathrm{CM}$ frame threshold, so that $\chi^{2}=\epsilon \omega(1-\cos \Theta) / 2 \sim \epsilon \omega \theta_{m}^{2} / 4 \geq 1$. Photons of energy $\epsilon$ then preferentially collide with those of energy $\omega \sim 4 /\left(\epsilon \theta_{m}^{2}\right)$ so that a $\epsilon^{-(1+\alpha)}$ spectrum implies the proportionality in equation (5).

The range of $\theta_{m} \ll 1$ is the most interesting for gamma-ray bursts of extragalactic origin, corresponding to a relative Lorentz factor between the comoving plasma frame and the observer's frame of $\gamma_{B} \sim 1 / \theta_{m} \gg 1$. Optical depths for truncated power-law spectra were presented by Gould \& Schreder (1967) and Krolik \& Pier (1991). Low-energy cutoffs would be at energies $\epsilon_{c}$ below $20 \mathrm{keV}$ for GRB spectra, thereby modifying the determination of the optical depth given here only above $\sim 12 \mathrm{MeV}$ for isotropic radiation, or at much higher energies $\epsilon \sim \gamma_{B}^{2} / \epsilon_{c}$ for strongly beamed photons. Such cutoffs would inhibit the $\gamma \gamma \rightarrow e^{+} e^{-}$attenuation of the continuum, however they are unlikely to be influential for the range of photon energies generally observed in bursts if $\gamma_{B} \gg 1$. In analyses where consideration of low-energy cutoffs is required, the optical depth takes the form of equation (5) but with the substitution

$$
\begin{aligned}
\mathscr{H}(\alpha) \rightarrow \mathscr{H}(\alpha, \eta)=\frac{4}{\sigma_{T}} \int_{\eta}^{\infty} d \chi \chi^{-(1+2 \alpha)} \sigma_{\gamma \gamma}(\chi), & \\
\eta & =\max \left\{1, \epsilon_{c} \epsilon \theta_{m}^{2} / 4\right\},
\end{aligned}
$$

for $\theta_{m} \ll 1$, using equations (21-23) of Gould \& Schreder (1967).

\section{RELATIVISTIC BEAMING IN GAMMA-RAY BURSTS}

Given the dependence in equation (5), the variation of the pair production turnover energy with beaming angle $\theta_{m}$ can now be simply obtained. A source of "size" $R$ that is a distance $d$ from Earth and has a density $n_{\gamma}$ of photons (all measured in the observer's frame), yields a flux $f_{\text {obs }}$ observed at Earth of

$$
f_{\text {obs }}=4 \pi n_{\gamma} c \frac{R^{2}}{d^{2}} .
$$

Since reference to the minimum photon energy observed is omitted here (i.e., infinite power-law spectra are considered), $n_{\gamma}$ and $f_{\text {obs }}$ represent the photon density and flux (per $m_{e} c^{2}$ ) at $\epsilon=1$. The size $R$ differs from the variability size $R_{v}\left(\sim 10^{6} \mathrm{~cm}\right.$ for GRBs) of the emission region, which is obtained from the variability timescale. For highly beamed photons, variability can be much more rapid than for isotropic photons, yielding small source variability sizes:

$$
R_{v} \sim R \theta_{m}, \quad \theta_{m} \ll 1
$$

Hence $R \sim \gamma_{B} R_{v}$, and emission regions much larger than neutron stars can have the same variability timescales if their radiation is highly beamed.

Equations (5), (12)-(14) can be pieced together to yield $\tau_{\gamma \gamma}(\epsilon) \propto \theta_{m}^{3+2 \alpha} \epsilon^{\alpha}$, which is equivalent to the result in equation (6) of Krolik \& Pier (1991), who determined the optical depth in the comoving plasma frame and then boosted to the observer's frame. If the spectrum is observed to break at an energy $\epsilon_{B}$, where $\tau_{\gamma \gamma}\left(\epsilon_{B}\right)=1$ in equation (5), then a constraint on the bulk Lorentz factor $\gamma_{B} \sim 1 / \theta_{m} \gg 1$ can be obtained for sources that are a distance $d$ from Earth and at redshift $z$ :

$$
\begin{aligned}
& \gamma_{B}^{3+2 \alpha} \sim \frac{39.2 f_{\mathrm{obs}}(1+z)^{\alpha}}{(1+\alpha)^{5 / 3}(7 / 3+\alpha)^{27 / 11}} \\
& \quad \times\left(\frac{d}{1 \mathrm{kpc}}\right)^{2}\left(\frac{R_{v}}{10^{6} \mathrm{~cm}}\right)^{-1} \epsilon_{B}^{\alpha} .
\end{aligned}
$$

This solution of $\tau_{\gamma \gamma}\left(\epsilon_{B}\right)=1$ is for $\int_{\text {obs }}$ in $\mathrm{cm}^{-2} \mathrm{~s}^{-1}\left(m_{e} c^{2}\right)^{-1}$ and is depicted in Figure 1 for $f_{\mathrm{obs}}=1 \mathrm{~cm}^{-2} \mathrm{~s}^{-1}\left(m_{e} c^{2}\right)^{-1}$ and $R_{v}=$ $10^{6} \mathrm{~cm}$.

Clearly the beaming can be extremely relativistic, and the constraint in equation (15) is strongly dependent on the hard $\mathrm{X}$-ray spectral index of the source; these provide strong constraints for gamma-ray burst models. Specifically, typical bursts have hard X-ray spectral indices of $\alpha \sim 0-1$, so that Figure 1 indicates that sources at distances of $\sim 1 \mathrm{Gpc}$ must typically be beamed with bulk Lorentz factors of $\sim 10^{3}-10^{4}$ (observational parameters for BATSE bursts with spectral 
breaks are tabulated in Baring 1993). The redshift factor $1+z$ slightly enhances the beaming in cosmological sources (at $d>$ $1 \mathrm{Gpc}$ ). Central to this result is the dependence $\theta_{m} \propto 1 / \gamma_{B}$, which naturally occurs in many systems where a quasi-isotropic radiation field arises in a local plasma frame that moves with a Lorentz factor $\gamma_{B}$ relative to the observer's frame. Relativistic kinematics guarantees this dependence and is applicable to radiation processes such as inverse Compton scattering and synchrotron emission.

The relativistic beaming implied by Figure 1 for extragalactic bursts naturally blueshifts the break to hard gamma-ray energies. In the source plasma frame, a spectral break due to $\gamma-\gamma$ attenuation must always occur above $\sim 0.5 \mathrm{MeV}$. This is because efficient spectral attenuation at lower energies produces a pair plasma of Thomson optical depth greater than unity (the pair and photon densities become comparable) so that then Comptonization will strongly modify the continuum, resulting in a Wien peak that is not observed in sources. In the observer's frame the breaks must therefore appear above about $\gamma_{B} m_{e} c^{2}$. This Comptonization constraint can be combined with the pair production constraint in equation (15) to yield a definitive lower bound to the bulk Lorentz factor in GRBs for $\gamma_{B} \gg 1$ :

$$
\begin{aligned}
\gamma_{B}^{3+\alpha} \gtrsim \frac{39.2 f_{\mathrm{obs}}(1+z)^{\alpha}}{(1+\alpha)^{5 / 3}(7 / 3+\alpha)^{27 / 11}} & \\
& \times\left(\frac{d}{1 \mathrm{kpc}}\right)^{2}\left(\frac{R_{v}}{10^{6} \mathrm{~cm}}\right)^{-1} .
\end{aligned}
$$

Again $f_{\text {obs }}$ is expressed in units of $\mathrm{cm}^{-2} \mathrm{~s}^{-1}\left(m_{e} c^{2}\right)^{-1}$. Lower $\gamma_{B}$ are not possible unless another radiation process dominates $\gamma \gamma \rightarrow e^{+} e^{-}$as a means of attenuating the spectrum at $\mathrm{MeV}$

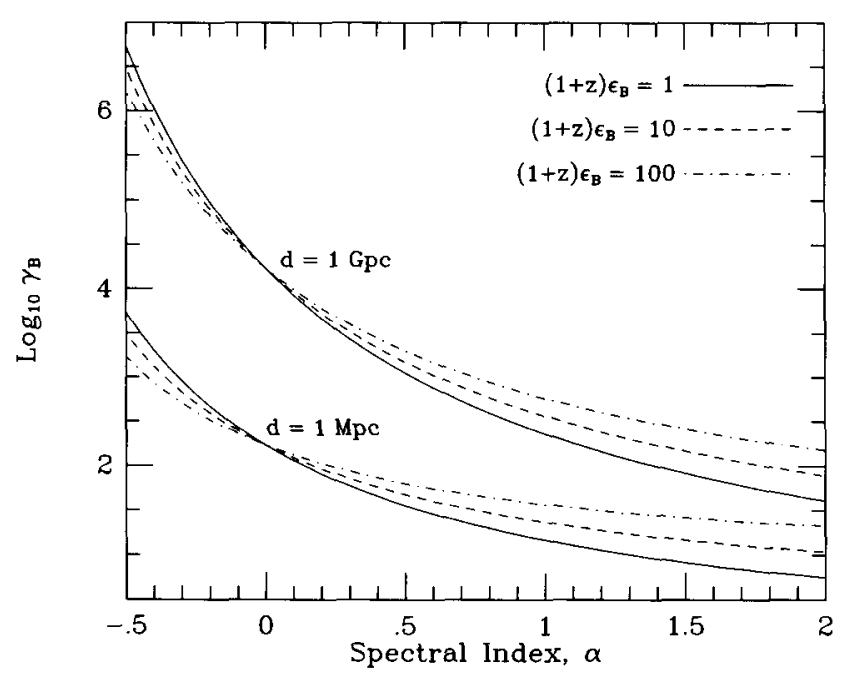

FIG. 1.-The variation of the bulk Lorentz factor $\gamma_{B} \sim 1 / \theta_{m}$ in GRBs (the beaming angle $\theta_{m}$ is defined in eq. [1]), as determined by the pair production constraint in eq. (15), with GRB spectral index $\alpha$ for powerlaw source spectra $n(\epsilon) \propto \epsilon^{-(1+\alpha)}$ that are observed to break at energies $\epsilon_{B}=$ 1,10 , and 100 (in units of $m_{e} c^{2}$ ). Results are displayed for different source distances $d$ as labeled, and are for $f_{\text {obs }}=1 \mathrm{~cm}^{-2} \mathrm{~s}^{-1}\left(m_{e} c^{2}\right)^{-1}$ and $R_{v}=10^{6}$ $\mathrm{cm}$. The GRB (cosmological) redshift $z$ ( $\approx 0$ for $d$ chosen) acts purely to scale the observed break energy.

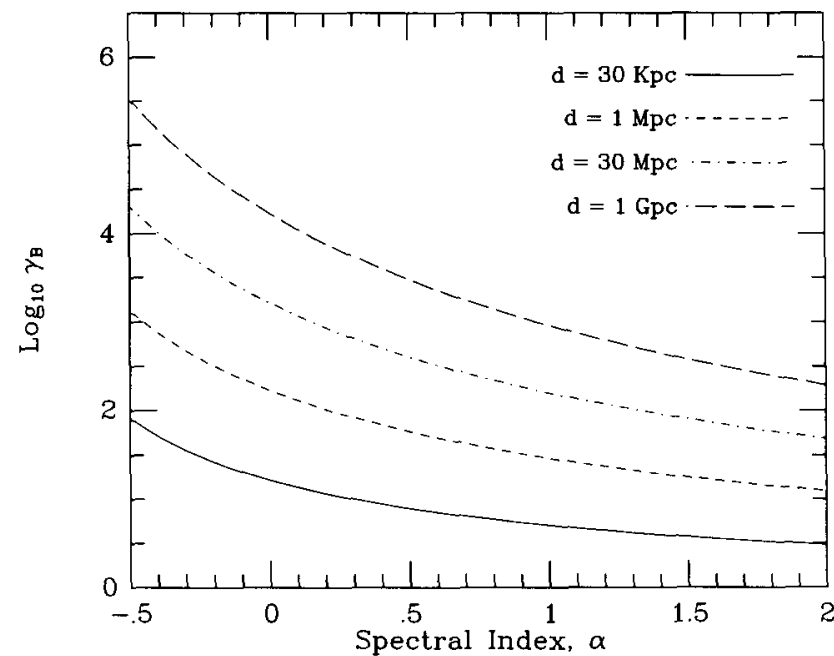

Fig. 2,-The absolute minimum bulk Lorentz factor $\gamma_{B} \sim 1 / \theta_{m}$ in GRBs, as determined by the pair production/Comptonization constraint in eq. (16), as a function of GRB spectral index $\alpha$ for power-law source spectra $n(\epsilon) \propto \epsilon^{-(1+\alpha)}$. The curves are lower bounds for $\gamma_{B}$ and are different source distances $d$ with $f_{\text {obs }}=1 \mathrm{~cm}^{-2} \mathrm{~s}^{-1}\left(m_{p} c^{2}\right)^{-1}$ and $R_{v}=10^{6} \mathrm{~cm}$. Any pair production breaks must occur above an energy $\gamma_{B} m_{e} c^{2}$. Note that the redshift $z \approx 0$ for the values of $d$ chosen.

energies; magnetic pair production $\gamma \rightarrow e^{+} e^{-}$in the strong fields of neutron stars is an example of such a process. Figure 2 displays the constraint in equation (16) for $f_{\text {obs }}=1 \mathrm{~cm}^{-2} \mathrm{~s}^{-1}$ $\left(m_{e} c^{2}\right)^{-1}$ and $R_{v}=10^{6} \mathrm{~cm}$. Fine-scale modifications to equation (16) can be made using model-dependent pair cascade calculations. Note that for cosmological sources the distance $d$ and the redshift $z$ in equations (15) and (16) can be related by standard cosmological expansion formulae (e.g., see eqs. 15.3.22-15.3.23 of Weinberg 1972).

Most bursts observed by the BATSE experiment have no distinguishable spectral breaks, so that the inferred large Lorentz factors are inconsequential, placing no severe demands on the hypothesis that they are cosmological in origin. However, the condition $\epsilon_{B} \gtrsim \gamma_{B}$ (the absence of spectral Comptonization) implies that there is only moderate beaming for those sources with breaks observed below $10 \mathrm{MeV}$, if indeed pair production is the mechanism for attenuation. It follows that the sources with observed breaks are either not cosmological, or that $\gamma \gamma \rightarrow e^{+} e^{-}$is not responsible for their spectral breaks.

\section{DISCUSSION}

The constraints in equations (15) and (16) demand that (1) extragalactic bursts have relativistic beaming and (2) a cosmological interpretation of GRBs requires emission regions with bulk Lorentz factors $\gamma_{B} \sim 1 / \theta_{m}$ far in excess of those in extragalactic jets, unless the GRB spectrum is steep $(\alpha \gtrsim 2)$. This evidence for seemingly incredible bulk acceleration for an astrophysical source, is actually quite compatible with fireball models for bursts (Cavallo \& Rees 1978; Paczyński 1986; Rees \& Mészáros 1992; Mészáros \& Rees 1992), where the huge energy of a cosmological burst drives a highly relativistic ( and optically thick) wind with $\gamma_{B} \sim 10^{3}$. In this context, $R$ would define the size of the outer layers of the fireball where $\tau_{\gamma \gamma} \sim 1$.

A necessary requirement that such bulk acceleration occurs 
is that in the earlier stages of the fireball (1) the temperature is relativistic, and (2) the expansion timescale is shorter than the (local) cooling timescale so that large expansion anisotropies may build before the expanding fireball cools to mildly relativistic temperatures. The first requirement has spawned truly exotic mechanisms for the initiation of these unimagineably powerful explosions (e.g., Eichler et al. 1989). Fireballs may generate nonthermal spectra in their outer layers (Cavallo \& Rees 1978), which may be affected in a multitemperature environment or if burst emission is scattered by external regions (Paczyński 1986; Rees \& Mészáros 1992). The correct prediction of GRB spectral breaks is a crucial test of any detailed fireball model for bursts and should be a focus of future theoretical work.

If the BATSE sources with breaks observed in the MeV energy range are extragalactic in origin, a mechanism other than $\gamma \gamma \rightarrow e^{+} e^{-}$probably generates their breaks. A few possibilities for such a mechanism were discussed by Baring (1993), who concluded that it would be difficult to find a single model that would make successful predictions of the spectra of all of the $G R O$ sources with MeV breaks. Spectral breaks can be created by breaks in the distribution function for the electrons that generate the radiation. This can occur in environments where relativistic electrons only partially cool, not reaching thermal equilibrium; i.e., the cooling time $t_{c}$ exceeds the timescale $t_{\mathrm{esc}}$ for radiation escape from the emission region. For cooling by the inverse Compton or synchrotron processes, a power-law population of electrons $\gamma^{-p}$ cools above some critical Lorentz factor $\gamma_{c}$, which depends on the energy density of soft photons or the magnetic field, to a steeper form $\gamma^{-(1+p)}$ (e.g., Lightman
\& Zdziarski 1987). This yields a break of $1 / 2$ in the photon spectrum, which turns out to be fine for BATSE source GRB 910503 but not for the other BATSE bursts with breaks (see Baring 1993, for observational parameters). This mechanism for break production is extremely model dependent, perhaps generating a large range of possible break energies, and is applicable to either a Galactic (+halo) or cosmological origin of bursts; in general it can be a cause of only small to moderate breaks.

The results derived in this paper indicate that the presence or absence of a spectral break can be used as a diagnostic on the radiation beaming in individual GRBs if they are at cosmological distances or otherwise. Equation (16) represents a definitive lower limit to $\gamma_{B}$ in a burst, if its spectrum is subject to pair production attenuation and Comptonization of the continuum does not occur. It appears that it may be difficult to find one model that accurately predicts spectra for all sources with observed $\mathrm{MeV}$ breaks. This provides a challenge for future theoretical work. In addition, beaming constraints on theoretical models imply that spatial transport in the source and the spectral index of the radiation will be intimately related. Determination of the radiation mechanism(s) operating in GRBs with breaks will provide valuable clues to the extent of particle acceleration that occurs in the sources, whether or not it corresponds relativistic bulk motion, and ultimately to the origin of gamma-ray bursts.

I thank M. Rees, A. Harding and A. Mastichiadis for helpful comments, and B. Dingus for alerting me to the latest EGRET spectral results on GRBs.
Baring, M. G. 1993, ApJ, 90, 899

Cavallo, G., \& Rees, M. J. 1978, MNRAS, 183, 359

Eichler, D., Livio, M., Piran, T., \& Schramm, D. N. 1989, Nature, 340, 126

Fenimore, E. E., Epstein, R. E., \& Ho, C. 1992, in AIP Conf. Proc. 265, Gamma-Ray Bursts, ed. W. S. Paciesas \& G. J. Fishman (New York: AIP), 158

Gould, R. J., \& Schreder, G. P. 1967, Phys. Rev., 155, 1404

Gradshteyn, I. S., \& Ryzhik, I. M. 1980, Table of Integrals, Series and Products (New York: Academic)

Kouveliotou, C, et al. 1994, ApJL, submitted

Krolik, J. H., \& Pier, E. A. 1991, ApJ, 373, 277

Kwok, P. W., et al. 1993, in Proc. Compton Observatory Symposium (St. Louis, MO) (New York: AIP), in press

\section{REFERENCES}

Lightman, A. P., \& Zdziarski, A. A. 1987, ApJ, 319, 643

Mao, S., \& Paczyński, B. 1992a, ApJ, 388, L45

1992b, ApJ, 389, L13

Meegan, C.. et al. 1992, Nature, 355, 143

Mészáros, P., \& Rees, M. J. 1992, MNRAS, 257, 29P

Paczyński, B. 1986, ApJ, 308, L43

Rees, M. J., \& Mészáros, P. 1992, MNRAS, 258, 41P

Schaefer, B. E., et al. 1992, ApJ, 393, L51

Schmidt, W. K. H. 1978, Nature, 271, 525

Schneid, E. J. 1992, A\&A, 255, L13

Sommer, M., et al. 1994, ApJL, in press

Stepney, S., \& Guilbert, P. W. 1983, MNRAS, 204, 1269

Svensson, R. 1987, MNRAS, 227, 403

Weinberg, S. 1972, Gravitation and Cosmology (New York: Wiley) 